\title{
On the regularity of the solution for the second initial boundary value problem for hyperbolic systems in domains with conical points
}

\author{
Nguyen Manh Hung ${ }^{1}$, Nguyen Thanh Anh $^{{ }^{*}}$ and Phung Kim Chuc ${ }^{2}$
}

\author{
* Correspondence: \\ thanhanh@hnue.edu.vn \\ 'Department of Mathematics, \\ Hanoi National University of \\ Education, Hanoi, Vietnam \\ Full list of author information is \\ available at the end of the article
}

\author{
Abstract \\ In this paper, we deal with the second initial boundary value problem for higher \\ order hyperbolic systems in domains with conical points. We establish several results \\ on the well-posedness and the regularity of solutions.
}

\section{Introduction}

Boundary value problems in nonsmooth domains have been studied in differential aspects. Up to now, elliptic boundary value problems in domains with point singularities have been thoroughly investigated (see, e.g, [1,2] and the extensive bibliography in this book). We are concerned with initial boundary value problems for hyperbolic equations and systems in domains with conical points. These problems with the Dirichlet boundary conditions were investigated in [3-5] in which the unique existence, the regularity and the asymptotic behaviour near the conical points of the solutions are established. The Neumann boundary problem for general second-order hyperbolic systems with the coefficients independent of time in domains with conical points was studied in [6]. In the present paper we consider the Cauchy-Neumann (the second initial) boundary value problem for higher-order strongly hyperbolic systems in domains with conical points.

Our paper is organized as follows. Section 2 is devoted to some notations and the formulation of the problem. In Section 3 we present the results on the unique existence and the regularity in time of the generalized solution. The global regularity of the solution is dealt with in Section 4.

\section{Notations and the formulation of the problem}

Let $\Omega$ be a bounded domain in $\mathbb{R}^{n}, n \geq 2$, with the boundary $\partial \Omega$. We suppose that $\partial \Omega$ is an infinitely differentiable surface everywhere except the origin, in a neighborhood of which $\Omega$ coincides with the cone $K=\{x: x /|x| \in G\}$, where $G$ is a smooth domain on the unit sphere $S^{n-1}$. For each $t, 0<t \leq \infty$, denote $Q_{t}=\Omega \times(0, \mathrm{t}), \Omega_{t}=\Omega \times\{\mathrm{t}\}$. Especially, we set $Q=Q_{\infty}, \Gamma=\partial \Omega \backslash\{0\}, S=\Gamma \times[0,+\infty)$.

(c) 2011 Hung et al; licensee Springer. This is an Open Access article distributed under the terms of the Creative Commons Attribution License (http://creativecommons.org/licenses/by/2.0), which permits unrestricted use, distribution, and reproduction in any medium, provided the original work is properly cited. 
For each multi-index $p=\left(p_{1}, \ldots, p_{n}\right) \in \mathbb{N}^{n}$, we use notations $|p|=p_{1}+\ldots+p_{n}$, $D^{p}=\frac{\partial^{|p|}}{\partial x_{1}^{p_{1}} \ldots \partial x_{n}^{p_{n}}}$. For a complex-valued vector function $u=\left(u_{1}, \ldots, u_{s}\right)$ defined on $Q$, we denote $D_{u}^{p}=\left(D_{u 1^{1}}^{p}, \ldots, D_{u s}^{p}\right), u_{t j}=\frac{\partial^{j} u}{\partial t^{j}}=\left(\frac{\partial^{j} u_{1}}{\partial t^{j}}, \ldots, \frac{\partial^{j} u_{s}}{\partial t^{j}}\right),|u|=\left(\sum_{j=1}^{s}\left|u_{j}\right|^{2}\right)^{\frac{1}{2}}$ :

Let us introduce the following functional spaces used in this paper. Let $l$ denote a nonnegative integer.

$H^{l}(\Omega)$ - the usual Sobolev space of vector functions $u$ defined in $\Omega$ with the norm

$$
\|u\|_{H^{\prime}(\Omega)}=\left(\int_{\Omega} \sum_{|p| \leq l}\left|D^{p} u\right|^{2} d x\right)^{\frac{1}{2}}<\infty .
$$

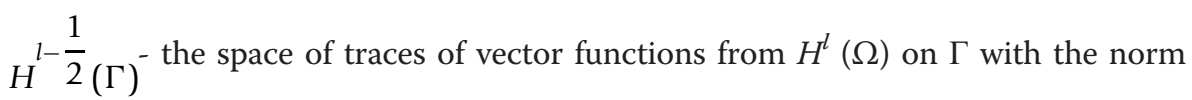

$$
\|u\|_{H^{l-\frac{1}{2}(\Gamma)}}=\inf \left\{\|v\|_{H^{l}(\Omega)}: v \in H^{l}(\Omega),\left.v\right|_{\Gamma}=u\right\} .
$$

$H^{l, 0}(Q, \gamma)(\gamma \in \mathbb{R})$ - the weighted Sobolev space of vector functions $u$ defined in $Q$ with the norm

$$
\|u\|_{H^{l, 0}(Q, \gamma)}=\left(\int_{Q} \sum_{|p| \leq l}\left|D^{p} u\right|^{2} e^{-2 \gamma t} d x d t\right)^{\frac{1}{2}}<\infty
$$

Especially, we set $L_{2}(Q, \gamma)=H^{0,0}(Q, \gamma)$.

$H^{l, 1}(Q, \gamma)(\gamma \in \mathbb{R})$ - the weighted Sobolev space of vector functions $u$ defined in $Q$ with the norm

$$
\|u\|_{H^{l, 1}(Q, \gamma)}=\left(\int_{Q}\left(\sum_{|p| \leq l}\left|D^{p} u\right|^{2}+\left|u_{t}\right|^{2}\right) e^{-2 \gamma t} d x d t\right)^{\frac{1}{2}}<\infty .
$$

$V_{2, \alpha}^{l}(\Omega)$ - the closure of $C_{0}^{\infty}(\bar{\Omega} \backslash\{0\})$ with respect to the norm

$$
\|u\|_{V_{2, \alpha}^{l}(\Omega)}=\left(\sum_{|p| \leq l} \int_{\Omega} r^{2(\alpha+|p|-l)}\left|D^{p} u\right|^{2} d x\right)^{\frac{1}{2}},
$$

where $r=|x|=\left(\sum_{k=1}^{n} x_{k}^{2}\right)^{\frac{1}{2}}$.

$H_{\alpha}^{l}(\Omega)(\alpha \in \mathbb{R})$-the weighted Sobolev space of vector functions $u$ defined in $\Omega$ with the norm

$$
\|u\|_{H_{\alpha}^{l}(\Omega)}=\left(\sum_{|p| \leq l} \int_{\Omega} r^{2 \alpha}\left|D^{p} u\right|^{2} d x\right)^{\frac{1}{2}}
$$


If $l \geq 1$, then $V_{\alpha}^{l-\frac{1}{2}}(\Gamma), H_{\alpha}^{l-\frac{1}{2}}(\Gamma)$ denote the spaces consisting of traces of functions from respective spaces $V_{2, \alpha}^{l}(\Omega), H_{\alpha}^{l}(\Omega)$ on the boundary $\Gamma$ with the respective norms

$$
\begin{aligned}
& \|u\|_{V_{\alpha}^{l-\frac{1}{2}}(\Gamma)}=\inf \left\{\|v\|_{V_{2, \alpha}^{l}(\Omega)}: v \in V_{2, \alpha}^{l}(\Omega),\left.v\right|_{\Gamma}=u\right\}, \\
& \|u\|_{H_{\alpha}^{l-\frac{1}{2}}(\Gamma)}=\inf \left\{\|v\|_{H_{\alpha}^{l}(\Omega)}: v \in H_{\alpha}^{l}(\Omega),\left.v\right|_{\Gamma}=u\right\} .
\end{aligned}
$$

$H_{\alpha}^{l, 1}(Q, \gamma)(\alpha, \gamma \in \mathbb{R})$ - the weighted Sobolev space of vector functions $u$ defined in $Q$ with the norm

$$
\|u\|_{H_{\alpha}^{l, 1}(Q, \gamma)}=\left(\int_{Q}\left(\sum_{|p| \leq l} r^{2 \alpha}\left|D^{p} u\right|^{2}+\left|u_{t}\right|^{2}\right) e^{-2 \gamma t} d x d t\right)^{\frac{1}{2}}<\infty
$$

From the definitions it follows the continuous imbeddings

$$
V_{2, \alpha}^{l}(\Omega) \subset H_{\alpha}^{l}(\Omega)
$$

and

$$
V_{2, \alpha+k}^{l+k}(\Omega) \subset V_{2, \alpha}^{l}(\Omega)
$$

for arbitrary nonnegative integers $l, k$ and real number $\alpha$. It is also well known (see [[2], Th. 7.1.1]) that if $\alpha<-\frac{n}{2}$ or $\alpha>l-\frac{n}{2}$ then

$$
V_{2, \alpha}^{l}(\Omega) \equiv H_{\alpha}^{l}(\Omega)
$$

with the norms being equivalent.

Now we introduce the differential operator

$$
L u=L(x, t, D) u=\sum_{|p|,|q| \leq m}(-1)^{|p|} D^{p}\left(a_{p q} D^{q} u\right),
$$

where $a_{p q}=a_{p q}(x, t)$ are the $s \times s$ matrices with the bounded complex-valued components in $\bar{Q}$. We assume that $a_{p q}=(-1)^{|p|+|q|} a_{q p}^{*}$ for all $|p|,|q| \leq m$, where $a_{q p}^{*}$ is the transposed conjugate matrix to $a_{p q}$. This means the differential operator $L$ is formally self-adjoint. We assume further that there exists a positive constant $\mu$ such that

$$
\sum_{|p|=|q|=m} a_{p q}(x, t) \eta_{q} \overline{\eta_{p}} \geq \mu \sum_{|p|=m}\left|\eta_{p}\right|^{2}
$$

for all $\eta_{p} \in \mathbb{C}^{s},|p|=m$, and all $(x, t) \in \bar{Q}$.

Let $v$ be the unit exterior normal to $S$. It is well known that (see, e.g., [[7], Th. 9.47]) there are boundary operators $N_{j}=N_{j}(x, t, D), j=1,2, \ldots, m$ on $S$ such that integration equality

$$
\int_{\Omega} L u \bar{v} d x=\sum_{|p|,|q| \leq m} \int_{\Omega} a_{p q} D^{q} u \overline{D^{p} v} d x+\sum_{j=1}^{m} \int_{\partial \Omega} N_{j} u \frac{\overline{\partial^{j-1} v}}{\partial v^{j-1}} d s
$$

holds for all $u, v \in C^{\infty}(\bar{\Omega})$ and for all $t \in[0, \infty)$. The order of the operator $N_{j}$ is $2 m$ $j$ for $j=1,2, \ldots, m$. 
In this paper, we consider the following problem:

$$
\begin{aligned}
& u_{t t}+L u=f \text { in } Q, \\
& N_{j} u=0 \text { on } S, j=1, \ldots, m, \\
& \left.u\right|_{t=0}=\left.u_{t}\right|_{t=0}=0 \quad \text { on } \Omega .
\end{aligned}
$$

A complex vector-valued function $u \in H^{m, 1}(Q, \gamma)$ is called a generalized solution of problem (2.6)-(2.8) if and only if $\left.u\right|_{t=0}=0$ and the equality

$$
\int_{Q} u_{t} \bar{\eta}_{t} d x d t+\sum_{|p|,|q| \leq m} \int_{Q} a_{p q} D^{q} u \overline{D^{p} \eta} d x d t=\int_{Q} f \bar{\eta} d x d t
$$

holds for all $\eta(x, t) \in H^{m, 1}(Q)$ satisfying $\eta(x, t)=0$ for all $t \geq T$ for some positive real number $T$.

\section{The unique solvability and the regularity in time}

First, we introduce some notations which will be used in the proof of Theorems 3.3 and 3.4. For each vector function $u, v$ defined in $\Omega$ and each nonnegative integer $k$,

$$
|u|_{k, \Omega}=\left(\int_{\Omega} \sum_{|p|=k}\left|D^{p} u\right|^{2} d x\right)^{\frac{1}{2}}, \quad(u, v)_{\Omega}=\int_{\Omega} u \bar{v} d x .
$$

For vector functions $u$ and $v$ defined in $Q$ and $\tau>0$, we set

$$
\begin{aligned}
& |u|_{k, Q_{\tau}}=\left(\int_{0}^{\tau}|u(\cdot, t)|_{k, \Omega}^{2} d t\right)^{\frac{1}{2}}, \quad|u|_{k, \Omega_{\tau}}=|u(\cdot, \tau)|_{k, \Omega,} \quad(u, v)_{\Omega_{\tau}}=(u(\cdot, \tau), v(\cdot, \tau))_{\Omega}, \\
& B_{t k}(t, u, v)=\sum_{|p|,|q| \leq m} \int_{\Omega} \frac{\partial^{k} a_{p q}}{\partial t^{k}}(\cdot, t) D^{q} u(\cdot, t) \overline{D^{p} v(\cdot, t)} d x, \quad B_{{ }^{\prime} k}^{\tau}(u, v)=\int_{0}^{\tau} B_{t k}(t, u, v) d t .
\end{aligned}
$$

Especially, we set

$$
B(t, u, v)=B_{t 0}(t, u, v) \quad \text { and } \quad B^{\tau}(u, v)=B_{t}^{\tau}(u, v) .
$$

From the formally self-adjointness of the operator $L$, we see that

$$
\overline{B(\tau, u, v)}=B(\tau, v, u) \text {. }
$$

Next, we introduce the following Gronwall-Bellman and interpolation inequalities as two fundamental tools to establish the theorems on the unique existence and the regularity in time.

Lemma 3.1 ([8], Lemma 3.1) Assume $u, \alpha, \beta$ are real-valued continuous on an interval $[a, b], \beta$ is nonnegative and integrable on $[a, b], \alpha$ is nondecreasing satisfying

$$
u(\tau) \leq \alpha(\tau)+\int_{a}^{\tau} \beta(t) u(t) d t \quad \text { for all } a \leq \tau \leq b .
$$


Then

$$
u(\tau) \leq \alpha(\tau) \exp \left(\int_{a}^{\tau} \beta(t) d t\right) \quad \text { for all } a \leq \tau \leq b .
$$

From [[9], Th. 4.14], we have the following lemma.

Lemma 3.2. For each positive real number $\varepsilon$ and each integer $j, 0<j<m$, there exists a positive real number $C=C(\Omega, m, \varepsilon)$ which is dependent on only $\Omega, m$ and $\varepsilon$ such that the inequality

$$
|u|_{j, \Omega}^{2} \leq \varepsilon|u|_{m, \Omega}^{2}+C|u|_{0, \Omega}^{2}
$$

holds for all $u \in H^{m}(\Omega)$.

Now we state and prove the main theorems of this section.

Theorem 3.3. Let $h$ be a nonnegative integer. Assume that all the coefficients $a_{p q}$ together with their derivatives with respect to t are bounded on $\bar{Q}$. Then there exists a positive real number $\gamma_{0}$ such that for each $\gamma>\gamma_{0}$, if $f \in L_{2}(Q, \sigma)$ for some nonnegative real number $\sigma$, the problem (2.6)-(2.8) has a unique generalized solution $u$ in the space $H^{m, 1}(Q, \gamma+\sigma)$ and

$$
\|u\|_{H^{m, 1}(Q, \gamma+\sigma)}^{2} \leq C\|f\|_{L_{2}(Q, \sigma)^{\prime}}^{2}
$$

where $C$ is a constant independent of $u$ and $f$.

Proof. The uniqueness is proved by similar way as in [[4], Th. 3.2]. We omit the detail here. Now we prove the existence by Galerkin approximating method. Suppose $\left\{\varphi_{k}\right\}_{k=1}^{\infty}$ is an orthogonal basis of $H^{m}(\Omega)$ which is orthonormal in $L_{2}(\Omega)$. Put

$$
u^{N}(x, t)=\sum_{k=1}^{N} c_{k}^{N}(t) \varphi_{k}(x),
$$

where $\left(c_{k}^{N}(t)\right)_{k=1}^{N}$ are the solution of the system of the following ordinary differential equations of second order:

$$
\left(u_{t t}^{N}, \varphi_{l}\right)_{\Omega_{t}}+B\left(t, u^{N}, \varphi_{l}\right)=\left(f, \varphi_{l}\right)_{\Omega_{t}}, \quad l=1, \ldots, N,
$$

with the initial conditions

$$
c_{k}^{N}(0)=0, \quad \frac{d c_{k}^{N}}{d t}(0)=0, \quad k=1, \ldots, N .
$$

Let us multiply (3.5) by $\frac{d c_{k}^{N}(t)}{d} d t$, take the sum with respect to $l$ from 1 to $N$, and integrate the obtained equality with respect to $t$ from 0 to $\tau(0<\tau<\infty)$ to receive

$$
\left(u_{t t}^{N}, u_{t}^{N}\right)_{\Omega_{t}}+B\left(t, u^{N}, u_{t}^{N}\right)=\left(f, u_{t}^{N}\right)_{\Omega_{t}} .
$$

Now adding this equality to its complex conjugate, then using (3.1) and the integration by parts, we obtain

$$
\left|u_{t}^{N}\right|_{0, \Omega_{\tau}}^{2}+B\left(\tau, u^{N}, u^{N}\right)=B_{t}^{\tau}\left(u^{N}, u^{N}\right)+2 \operatorname{Re}\left(f, u_{t}^{N}\right)_{Q_{\tau}} .
$$

With noting that, for some positive real number $\rho$,

$$
\rho\left|u^{N}\right|_{0, \Omega_{\tau}}^{2}=2 \operatorname{Re} \rho\left(u^{N}, u_{t}^{N}\right)_{Q_{\tau}},
$$


we can rewrite (3.8) as follows

$$
\begin{aligned}
& \left|u_{t}^{N}\right|_{0, \Omega_{\tau}}^{2}+B_{0}\left(\tau, u^{N}, u^{N}\right)+\rho|u|_{0, \Omega_{\tau}}^{2}=B_{t}^{\tau}\left(u^{N}, u^{N}\right) \\
& -\sum_{\substack{|p|,|q| \leq m \\
|p|+|q|<2 m-1}} \int_{\Omega_{\tau}} a_{p q} D^{q} u^{N} \overline{D^{p} u^{N}} d x+2 \operatorname{Re} \rho\left(u^{N}, u_{t}^{N}\right)_{Q_{\tau}}+2 \operatorname{Re}\left(f, u_{t}^{N}\right)_{Q_{\tau}} .
\end{aligned}
$$

By (2.4), the left-hand side of (3.9) is greater than

$$
\left|u_{t}^{N}(\cdot, \tau)\right|_{0, \Omega}^{2}+\mu\left|u^{N}(\cdot, \tau)\right|_{m, \Omega}^{2}+\rho\|u(\cdot, \tau)\|_{0, \Omega}^{2} .
$$

We denote by $I, I I, I I I, I V$ the terms from the first, second, third, and forth, respectively, of the right-hand sides of (3.9). We will give estimations for these terms. Firstly, we separate $I$ into two terms

$$
\sum_{|p|=|q|=m} \int_{Q_{\tau}} \frac{\partial a_{p q}}{\partial t} D^{q} u^{N} \overline{D^{p} u^{N}} d x d t+\sum_{\substack{|p|,|q| \leq m \\|p|+|q| \leq 2 m-1}} \int_{Q_{\tau}} \frac{\partial a_{p q}}{\partial t} D^{q} u^{N} \overline{D^{p} u^{N}} d x d t \equiv I_{1}+I_{2} .
$$

Put

$$
\mu_{1}=\sup \left\{\left|\frac{\partial a_{p q}}{\partial t}(x, t)\right|:|p|=|q|=m,(x, t) \in \bar{Q}\right\} \quad \text { and } \quad m^{\prime}=\sum_{|p|=m} 1 .
$$

Then, by the Cauchy inequality, we have

$$
I_{1} \leq \mu_{1} \sum_{|p|=|q|=m} \frac{1}{2}\left(\left|D^{q} u^{N}\right|_{0, Q_{\tau}}^{2}+\left|D^{p} u^{N}\right|_{0, Q_{\tau}}^{2}\right) \leq m^{\prime} \mu_{1}\left|u^{N}\right|_{m, Q_{\tau}}^{2}
$$

By the Cauchy inequality and the interpolation inequality (3.3), for an arbitrary positive number $\varepsilon_{1}$, we have

$$
I_{2} \leq \varepsilon_{1}\left|u^{N}\right|_{m, Q_{\tau}}^{2}+C_{1}\left|u^{N}\right|_{0, Q_{\tau^{\prime}}}^{2}
$$

where $C_{1}=C_{1}\left(\varepsilon_{1}\right)$ is a nonnegative constant independent of $u^{N}, f$ and $\tau$. Now using again the Cauchy and interpolation inequalities, for an arbitrary positive number $\varepsilon_{2}$ with $\varepsilon_{2}<\mu$, it holds that

$$
I I \leq \varepsilon_{2}\left|u^{N}(\cdot, \tau)\right|_{m, \Omega}^{2}+C_{2}\left|u^{N}(\cdot, \tau)\right|_{0, \Omega^{\prime}}^{2}
$$

where $C_{2}=C_{2}\left(\varepsilon_{2}\right)$ is a nonnegative constant independent of $u^{N}, f$ and $\tau$. For the terms $I I I$ and $I V$, by the Cauchy inequality, we have

$$
I I I \leq \frac{\left(\mu-\varepsilon_{2}\right) \rho^{2}}{m^{\prime} \mu_{1}+\varepsilon_{1}}\left|u^{N}\right|_{0, Q_{\tau}}^{2}+\frac{m^{\prime} \mu_{1}+\varepsilon_{1}}{\mu-\varepsilon_{2}}\left|u_{t}^{N}\right|_{0, Q_{\tau^{\prime}}}^{2}
$$

and

$$
I V \leq \varepsilon_{3}\left|u_{t}^{N}\right|_{0, Q_{\tau}}^{2}+\frac{1}{\varepsilon_{3}}|f|_{0, Q_{\tau}}^{2}
$$


where $\varepsilon_{3}>0$, arbitrary. Combining the above estimations we get from (3.9) that

$$
\begin{array}{r}
\left|u_{t}^{N}(\cdot, \tau)\right|_{0, \Omega}^{2}+\left(\mu-\varepsilon_{2}\right)\left|u^{N}(\cdot, \tau)\right|_{m, \Omega}^{2}+\left(\rho-C_{2}\right)\left|u^{N}(\cdot, \tau)\right|_{0, \Omega}^{2} \leq\left(m^{\prime} \mu_{1}+\varepsilon_{1}\right)\left|u^{N}\right|_{m, Q_{\tau}}^{2} \\
+\left(C_{1}+\frac{\left(\mu-\varepsilon_{2}\right) \rho^{2}}{m^{\prime} \mu_{1}+\varepsilon_{1}}\right)\left|u^{N}\right|_{0, Q_{\tau}}^{2}+\left(\frac{m^{\prime} \mu_{1}+\varepsilon_{1}}{\mu-\varepsilon_{2}}+\varepsilon_{3}\right)\left|u_{t}^{N}\right|_{0, Q_{\tau}}^{2}+\frac{1}{\varepsilon_{3}}|f|_{0, Q_{\tau}}^{2} .
\end{array}
$$

Now fix $\varepsilon_{1}, \varepsilon_{2}$ and consider the function

$$
g(\rho)=\frac{C_{1}+\frac{\left(\mu-\varepsilon_{2}\right) \rho^{2}}{m^{\prime} \mu_{1}+\varepsilon_{1}}}{\rho-C_{2}} \text { for } \rho>C_{2} .
$$

We have

$$
\frac{d g}{d \rho}=\frac{\rho^{2}-2 C_{2} \rho-\frac{C_{1}}{A}}{A\left(\rho-C_{2}\right)^{2}} \quad \text { with } A=\frac{\left(\mu-\varepsilon_{2}\right) \rho^{2}}{m^{\prime} \mu_{1}+\varepsilon_{1}} .
$$

We see that the function $g$ has a unique minimum at

$$
\rho_{0}=\rho_{0}\left(\varepsilon_{1}, \varepsilon_{2}\right)=C_{2}+\sqrt{C_{2}^{2}+\frac{C_{1}}{A}} .
$$

We put

$$
\gamma_{0}=\frac{1}{2} \inf _{\substack{\varepsilon_{1}>0 \\ 0<\varepsilon_{2}<\mu}} \max \left\{\frac{m^{\prime} \mu_{1}+\varepsilon_{1}}{\mu-\varepsilon_{2}}, g\left(\rho_{0}\right)\right\} .
$$

Now we take real numbers $\gamma, \gamma_{1}$ arbitrarily satisfying $\gamma_{0}<\gamma_{1}<\gamma$. Then there are positive real numbers $\varepsilon_{1}, \varepsilon_{2},\left(\varepsilon_{2}<\mu\right), \rho\left(\rho>C_{2}\left(\varepsilon_{1}, \varepsilon_{2}\right)\right)$ and $\varepsilon_{3}$ such that

$$
\frac{m^{\prime} \mu_{1}+\varepsilon_{1}}{\mu-\varepsilon_{2}}+\varepsilon_{3}<2 \gamma_{1} \quad \text { and } \frac{C_{1}\left(\varepsilon_{1}, \varepsilon_{2}\right)+\frac{\left(\mu-\varepsilon_{2}\right) \rho^{2}}{m^{\prime} \mu_{1}+\varepsilon_{1}}}{\rho-C_{2}\left(\varepsilon_{1}, \varepsilon_{2}\right)}<2 \gamma_{1} \text {. }
$$

From now to the end of the present proof, we fix such constants $\varepsilon_{1}, \varepsilon_{2}, \varepsilon_{3}$ and $\rho$. Let $\||| u^{N}(\cdot, \tau)_{\Omega}^{2}|| \mid$ stand for the left-hand side of (3.10). It follows from (3.10) and (3.12) that

$$
\left\|\left|u^{N}(\cdot, \tau)\right|\right\|_{\Omega}^{2} \leq 2 \gamma_{1} \int_{0}^{\tau}\left|\left\|\left.u(\cdot, t)\left|\|_{\Omega}^{2} d t+C \int_{0}^{\tau}\right| f(\cdot, t)\right|_{0, \Omega} ^{2} d t \quad \text { for all } \tau \leq 0,\right.\right.
$$

where $C=\frac{1}{\varepsilon_{3}}$. By the Gronwall-Bellman inequality (3.2), we receive from (3.13) that

$$
\left.\left\|u^{N}(\cdot, \tau)\right\|\right|_{\Omega} ^{2} \leq C e^{2 \gamma_{1} \tau} \int_{0}^{\tau}|f(\cdot, t)|_{0, \Omega}^{2} d t \quad \text { for all } \tau \geq 0
$$

We see that

$$
\int_{0}^{\tau}|f(\cdot, t)|_{0, \Omega}^{2} d t=e^{2 \sigma \tau} \int_{0}^{\tau}\left|e^{-\sigma \tau} f(\cdot, t)\right|_{0, \Omega}^{2} d t \leq e^{2 \sigma \tau} \int_{0}^{\tau}\left|e^{-\sigma t} f(\cdot, t)\right|_{0, \Omega}^{2} d t .
$$

Hence, it follows from (3.14) that

$$
\|\| u^{N}(\cdot, \tau)\|\|_{\Omega}^{2} \leq C e^{2\left(\gamma_{1}+\sigma\right) \tau} \int_{0}^{\tau}\left|e^{-\sigma t} f(\cdot, t)\right|_{0, \Omega}^{2} d t \leq C e^{2\left(\gamma_{1}+\sigma\right) \tau}\|f\|_{L_{2}(Q, \sigma)}^{2} \quad \text { for } \tau \leq 0 .
$$


Now multiplying both sides of this inequality by $e^{-2(\gamma+\sigma) \tau}$, then integrating them with respect to $\tau$ from 0 to $\infty$, we arrive at

$$
\left\|\left|u^{N}\right|\right\|_{Q, \gamma+\sigma}^{2}:=\int_{0}^{\infty} e^{-2(\gamma+\sigma) \tau}\left\|\mid u^{N}(\cdot, \tau)\right\|\left\|_{\Omega}^{2} d \tau \leq C\right\| f \|_{L_{2}(Q, \sigma)}^{2} .
$$

It is clear that $\||\cdot|\|_{Q, \gamma+\sigma}$ is a norm in $H^{m, 1}(Q, \gamma+\sigma)$ which is equivalent to the norm $\|\cdot\|_{H^{m, 1}(Q, \gamma+\sigma)}$. Thus, it follows from (3.16) that

$$
\left\|u^{N}\right\|_{H^{m, 1}(Q, \gamma+\sigma)}^{2} \leq C\|f\|_{L_{2}(Q, \sigma)}^{2} .
$$

From this inequality, by standard weakly convergent arguments (see, e.g., [[10], Ch. 7]), we can conclude that the sequence $\left\{u^{N}\right\}_{N=1}^{\infty}$ possesses a subsequence convergent to a vector function $u \in H^{m, 1}(Q, \gamma+\sigma)$ which is a generalized solution of problem (2.6)(2.8). Moreover, it follows from (3.17) that the inequality (3.4) holds.

Theorem 3.4. Let $h$ be a nonnegative integer. Assume that all the coefficients $a_{p q}$ together with their derivatives with respect to $t$ up to the order $h$ are bounded on $\bar{Q}$. Let $\gamma_{0}$ be the number as in Theorem 3.3 which was defined by formula (3.11). Let the vector function $f$ satisfy the following conditions for some nonnegative real number $\sigma$

(i) $f_{t^{k}} \in L_{2}\left(Q, k \gamma_{0}+\sigma\right), k \leq h$,

(ii) $f_{t^{k}}(x, 0)=0,0 \leq k \leq h-1$.

Then for an arbitrary real number $\gamma$ satisfying $\gamma>\gamma_{0}$ the generalized solution $u$ in the space $H^{m, 1}(Q, \gamma+\sigma)$ of the problem (3.6)- (3.7) has derivatives with respect to $t$ up to the order $h$ with $u_{t^{k}} \in H^{m, 1}(Q,(k+1) \gamma+\sigma)$ for $k=0,1, \ldots, h$ and

$$
\sum_{k=0}^{h}\left\|u_{t^{k}}\right\|_{H^{m, 1}(Q,(k+1) \gamma+\sigma)}^{2} \leq C \sum_{k=0}^{h}\left\|f_{t^{k}}\right\|_{L_{2}\left(Q, k \gamma_{0}+\sigma\right)}^{2},
$$

where $C$ is a constant independent of $u$ and $f$.

Proof. From the assumptions on the regularities of the coefficients $a_{p q}$ and of the function $f$ it follows that the solution $\left(c_{k}^{N}(t)\right)_{k=1}^{N}$ of the system (3.5), (3.6) has generalized derivatives with respect to $t$ up to the order $h+2$. Now take an arbitrary real number $\gamma_{1}$ satisfying $\gamma_{0}<\gamma_{1}<\gamma$. We will prove by induction that

$$
\left\|u_{t^{k}}^{N}(\cdot, \tau)\right\|_{H^{m}(\Omega)}^{2} \leq C e^{2\left((k+1) \gamma_{1}+\sigma\right) \tau} \sum_{j=0}^{k}\left\|f_{t^{\prime}}\right\|_{L_{2}\left(Q, j \gamma_{0}+\sigma\right)}^{2} \quad \text { for } \tau>0
$$

and for $k=0, \ldots, h$, where the constant $C$ is independent of $N, f$ and $\tau$. From (3.15) it follows that (3.19) holds for $k=0$ since the norm $\||\cdot|\|$ is equivalent to the norm $\|\cdot\|_{H^{m}(\Omega)}$. Assuming by induction that (3.19) holds for $k=h-1$, we will show it to be true for $k=h$. To this end we differentiate $h$ times both sides of (3.5) with respect to $t$ to receive the following equality

$$
\left(u_{t^{h+2}}^{N}, \varphi_{l}\right)_{\Omega_{t}}+\sum_{k=0}^{h}\left(\begin{array}{l}
h \\
k
\end{array}\right) B_{t^{h-k}}\left(t, u_{t^{\prime}}^{N}, \varphi_{l}\right)=\left(f_{t^{h}}, \varphi_{l}\right)_{\Omega_{t}}, l=1, \ldots, N .
$$


From these equalities together with the initial (3.6) and the assumption (ii), we can show by induction on $h$ that

$$
\left.u_{t^{k}}^{N}\right|_{t=0}=0 \quad \text { for } k=0, \ldots, h+1 .
$$

Now multiplying both sides of (3.20) by $\frac{d^{h+1} c_{k}^{N}}{d} d t^{h+1}$, then taking sum with respect to $l$ from 1 to $N$, we get

$$
\left(u_{t^{h+2}}^{N}, u_{t^{h+1}}^{N}\right)_{\Omega_{t}}+\sum_{k=0}^{h}\left(\begin{array}{l}
h \\
k
\end{array}\right) B_{t^{h-k}}\left(t, u_{t^{k}}^{N}, u_{t^{h+1}}^{N}\right)=\left(f_{t^{h}}, u_{t^{h+1}}^{N}\right)_{\Omega_{t}} .
$$

Adding the equality (3.22) to its complex conjugate, we have

$$
\frac{\partial}{\partial t}\left|u_{t^{h+1}}^{N}\right|_{0, \Omega_{t}}^{2}+\sum_{k=0}^{h}\left(\begin{array}{l}
h \\
k
\end{array}\right)\left[\frac{\partial}{\partial t} B_{t^{h-k}}\left(t, u_{t^{k}}^{N}, u_{t^{h}}^{N}\right)-B_{t^{h-k+1}}\left(t, u_{t^{k}}^{N}, u_{t^{h}}^{N}\right)\right]=2 \operatorname{Re}\left(f_{t^{h}}, u_{t^{h+1}}^{N}\right)_{\Omega_{t}} .
$$

Integrating both sides of this equality with respect to $t$ from 0 to a positive real $\tau$ with using the integration by parts and (3.21), we arrive at

$$
\begin{aligned}
\left|u_{t^{h+1}}^{N}\right|_{0, \Omega_{\tau}}^{2}+B\left(\tau, u_{t^{h}}^{N}, u_{t^{h}}^{N}\right)=B^{\tau}\left(u_{t^{h}}^{N}, u_{t^{h}}^{N}\right)+\sum_{k=0}^{h-1}\left(\begin{array}{l}
h \\
k
\end{array}\right) B_{t^{h-k+1}}^{\tau}\left(u_{t^{k}}^{N}, u_{t^{h}}^{N}\right) \\
\quad-\sum_{k=0}^{h-1}\left(\begin{array}{l}
h \\
k
\end{array}\right) B_{t^{h-k}}\left(\tau, u_{t^{k}}^{N}, u_{t^{h}}^{N}\right)+2 \operatorname{Re}\left(f_{t^{h}}, u_{t^{h+1}}^{N}\right)_{Q_{\tau}} .
\end{aligned}
$$

This equality has the form (3.8) with $u^{N}$ replaced by $u_{t^{h}}^{N}$ and the last term of the righthand side of (3.8) replaced by the following expression

$$
\sum_{k=0}^{h-1}\left(\begin{array}{l}
h \\
k
\end{array}\right) B_{t^{h-k+1}}^{\tau}\left(u_{t^{k}}^{N}, u_{t^{h}}^{N}\right)-\sum_{k=0}^{h-1}\left(\begin{array}{l}
h \\
k
\end{array}\right) B_{t^{h-k}}\left(\tau, u_{t^{k}}^{N}, u_{t^{h}}^{N}\right)+2 \operatorname{Re}\left(f_{t^{h}}, u_{t^{h+1}}^{N}\right)_{Q_{\tau}} .
$$

Since the coefficients $a_{p q}$ together with their derivatives with respect to $t$ up to the order $h$ are bounded, by the Cauchy and interpolation inequalities and the induction assumption, we see that

$$
\begin{gathered}
\left|\sum_{k=0}^{h-1}\left(\begin{array}{l}
h \\
k
\end{array}\right) B_{t^{h-k}}\left(\tau, u_{t^{k}}^{N}, u_{t^{h}}^{N}\right)\right| \leq \varepsilon\left(\left|u_{t^{h}}^{N}(\cdot, \tau)\right|_{m, \Omega}^{2}+\left|u_{t^{h}}^{N}(\cdot, \tau)\right|_{0, \Omega}^{2}\right)+C \sum_{k=0}^{h-1}\left\|u_{t^{k}}^{N}(\cdot, \tau)\right\|_{m, \Omega_{\tau}}^{2} \\
\leq \varepsilon\left(\left|u_{t^{h}}^{N}(\cdot, \tau)\right|_{m, \Omega}^{2}+\left|u_{t^{h}}^{N}(\cdot, \tau)\right|_{0, \Omega}^{2}\right)+C e^{2\left(h \gamma_{1}+\sigma\right) \tau} \sum_{j=0}^{k}\left\|f_{t_{t}}\right\|_{L_{2}\left(Q, j \gamma_{0}+\sigma\right)^{\prime}}^{2} \\
\left|\sum_{k=0}^{h-1}\left(\begin{array}{l}
h \\
k
\end{array}\right) B_{t^{h}-k+1}^{\tau}\left(u_{t^{k^{\prime}}}^{N}, u_{t^{h}}^{N}\right)\right| \leq \varepsilon\left(\left|u_{t^{h}}^{N}\right|_{m, Q_{\tau}}^{2}+\left|u_{t^{h}}^{N}\right|_{0, Q_{\tau}}^{2}\right)+C \sum_{k=0}^{h-1}\left\|u_{t^{k}}^{N}\right\|_{m, Q_{\tau}}^{2} \\
=\varepsilon\left(\left|u_{t^{h}}^{N}\right|_{m, Q_{\tau}}^{2}+\left|u_{t^{h}}^{N}\right|_{0, Q_{\tau}}^{2}\right)+C \sum_{k=0}^{h-1} \int_{0}^{\tau}\left\|u_{t^{k}}^{N}(\cdot, t)\right\|_{m, \Omega}^{2} d t \\
\leq \varepsilon\left(\left|u_{t^{h}}^{N}\right|_{m, Q_{\tau}}^{2}+\left|u_{t^{h}}^{N}\right|_{0, Q_{\tau}}^{2}\right)+C \sum_{j=0}^{k}\left\|f_{t^{\prime}}\right\|_{L_{2}\left(Q, j \gamma_{0}+\sigma\right)}^{2} \int_{0}^{\tau} e^{2\left(h \gamma_{1}+\sigma\right) t} d t \\
\leq \varepsilon\left(\left|u_{t^{h}}^{N}\right|_{m, Q_{\tau}}^{2}+\left|u_{t^{h}}^{N}\right|_{0, Q_{\tau}}^{2}\right)+C e^{2\left(h \gamma_{1}+\sigma\right) \tau} \sum_{j=0}^{k}\left\|f_{t^{\prime}}\right\|_{L_{2}\left(Q, j \gamma_{0}+\sigma\right)}^{2} .
\end{gathered}
$$


and

$$
\begin{aligned}
\left|2 \operatorname{Re}\left(f_{t^{h}}, u_{t^{h+1}}^{N}\right)_{Q_{\tau}}\right| & \leq \varepsilon\left|u_{t^{h+1}}^{N}\right|_{0, Q_{\tau}}^{2}+C\left\|f_{t^{h}}\right\|_{L_{2}(Q)}^{2} \\
& \leq \varepsilon\left|u_{t^{h+1}}^{N}\right|_{0, Q_{\tau}}^{2}+C e^{2\left(h \gamma_{1}+\sigma\right) \tau}\left\|f_{t^{h}}\right\|_{L_{2}\left(Q, h \gamma_{0}+\sigma\right)}^{2} .
\end{aligned}
$$

Thus, repeating the arguments which were used to get (3.15) from (3.8), we can obtain (3.19) for $k=h$ from (3.23).

Now we multiply both sides of (3.19) by $e^{-2((k+1) \gamma+\sigma) \tau}$, then integrate them with respect to $\tau$ from 0 to $\infty$ to get

$$
\left\|u_{t^{k}}^{N}\right\|_{H^{m, 1}(Q,(k+1) \gamma+\sigma)}^{2} \leq C \sum_{j=0}^{k}\left\|f_{t^{j}}\right\|_{L_{2}\left(Q, j \gamma_{0}+\sigma\right)}^{2}, k=0, \ldots, h .
$$

From this inequality, by again standard weakly convergent arguments, we can conclude that the sequence $\left\{u_{t^{k}}^{N}\right\}_{N=1}^{\infty}$ possesses a subsequence convergent to a vector function $u^{(k)} \in H^{m, 1}(Q,(k+1) \gamma+\sigma)$, moreover, $u^{(k)}$ is the $k$ th generalized derivative in $t$ of the generalized solution $u$ of problem (2.6)-(2.8). The estimation (3.18) follows from (3.24) by passing the weak convergences.

\section{The global regularity}

First, we introduce the operator pencil associated with the problem. See [11] for more detail. For convenience we rewrite the operators $L(x, t, D), N_{j}(x, t, D)$ in the form

$$
\begin{aligned}
& L=L\left(x, t, \partial_{x}\right)=\sum_{|p| \leq 2 m} a_{p}(x, t) D^{p} \\
& N_{j}=N_{j}(x, t, D)=\sum_{|p| \leq 2 m-j} b_{j p}(x, t) D^{p}, \quad j=1, \ldots, m .
\end{aligned}
$$

Let $L_{0}(x, t, D), N_{0 j}(x, t, D)$, be the principal homogeneous parts of $L(x, t, D), N_{j}(x, t$, $D)$. It can be directly verified that the derivative $D^{\alpha}$ can be written in the form

$$
D^{\alpha}=r^{-|\alpha|} \sum_{p=0}^{|\alpha|} P_{\alpha, p}\left(\omega, D_{\omega}\right)\left(r D_{r}\right)^{p}
$$

where $P_{\alpha, p}\left(\omega, \partial_{\omega}\right)$ are differential operators of order $\leq|\alpha|-p$ with smooth coefficients on $\bar{\Omega}, r=|x|, \omega$ is an arbitrary local coordinate system on $S^{n-1}, D_{\omega}=\frac{\partial}{\partial \omega}$, $D_{r}=\frac{\partial}{\partial r}$. Thus we can write $L_{0}(0, t, D)$ and $N_{0 j}(0, t, D)$ in the form

$$
\begin{aligned}
& L_{0}(0, t, D)=r^{-2 m} \mathcal{L}\left(\omega, t, D_{\omega}, r D_{r}\right), \\
& N_{0, j}(0, t, D)=r^{-2 m+j} \mathcal{N}_{j}\left(\omega, t, D_{\omega}, r D_{r}\right) .
\end{aligned}
$$

The operator pencil associated with the problem is defined by

$$
\mathcal{U}(\lambda, t)=\left(\mathcal{L}\left(\omega, t, D_{\omega}, \lambda\right), \mathcal{N}_{j}\left(\omega, t, D_{\omega}, \lambda\right)\right), \lambda \in \mathbb{C}, t \in(0,+\infty) .
$$


For every fixed $\lambda \in \mathbb{C}$ and $t \in(0, \infty)$, the operator $\mathcal{U}(\lambda, t)$ continuously maps

$$
H^{2 m}(G) \text { into } L_{2}(G) \times \prod_{j=1}^{m} H^{j-\frac{1}{2}}(\partial G) .
$$

For some fixed $t \in(0, \infty)$, a complex number $\lambda_{0}$ is called an eigenvalue of $\mathcal{U}(\lambda, t)$ if there exists $\phi_{0} \in H^{2 m}(G)$ such that $\phi_{0} \neq 0$ and $\mathcal{U}\left(\lambda_{0}, t\right) \varphi_{0}=0$. It is well known that the spectrum of the operator $\mathcal{U}\left(\lambda_{0}, t\right)$ for each $t \in(0, \infty)$, is an enumerable set of eigenvalues (see [[2], Th. 5.2.1]).

Now let us give the main theorem of this section:

Theorem 4.1. Suppose that all the assumptions of Theorem 3.4 hold for a given positive integer $h$. Assume further that the strip

$$
m-\varepsilon-\frac{n}{2} \leq \operatorname{Re} \lambda \leq 2 m-\alpha-\frac{n}{2}
$$

does not contain any eigenvalue of $\mathcal{U}(\lambda, t)$ for all $t \in(0,+\infty)$ and for some real numbers $\varepsilon$ and $\alpha$ satisfying $0 \leq \alpha \leq m+\varepsilon, 0<\varepsilon<\frac{1}{2}$ if $m \geq \frac{n}{2}$ and $n$ is even, otherwise $\varepsilon=$ 0. Then $u_{t^{k}} \in H_{\alpha}^{2 m, 1}(Q,(k+2) \gamma+\sigma)$ for $k=0,1, \ldots, h-1$ and

$$
\sum_{k=0}^{h-1}\left\|u_{t^{k}}\right\|_{H_{\alpha}^{2 m, 1}(Q,(k+2) \gamma+\sigma)}^{2} \leq C \sum_{k=0}^{h}\left\|f_{t^{k}}\right\|_{L_{2}\left(Q, k \gamma_{0}+\sigma\right)}^{2},
$$

where $C$ is a constant independent of $u$ and $f$.

To prove Theorem 4.1 we need to establish some following lemmas.

Lemma 4.2. Let $l$ be a nonnegative integer, $t_{0}$ be a fixed number in $[0, \infty)$, and let $\left.u \in H_{l o c}^{l+2 m}(\bar{\Omega} \backslash\{0\})\right) \cap V_{2, \alpha-l-2 m}^{0}(\Omega)$ be a solution of the following elliptic boundary value problem

$$
\begin{aligned}
& L\left(x, t_{0}, D\right) u=f \quad \text { in } \Omega, \\
& N_{j}\left(x, t_{0}, D\right) u=g_{j} \quad \text { on } \Gamma, \quad j=1, \ldots, m,
\end{aligned}
$$

$$
\begin{aligned}
& \text { where } f \in V_{2, \alpha}^{l}(\Omega), g_{j} \in V_{2, \alpha}^{l+j-\frac{1}{2}}(\Gamma) \text {. Then } u \in V_{2, \alpha}^{l+2 m}(\Omega) \text { and the following estimate } \\
& \|u\|_{V_{2, \alpha}^{l+m}(\Omega)}^{2} \leq C\left(\|f\|_{V_{2, \alpha}^{l}(\Omega)}^{2}+\sum_{j=1}^{m}\left\|g_{j}\right\|_{\substack{l+j-\\
V_{2, \alpha}}}^{2}+\|u\|_{V_{2, \alpha-l-2 m}^{0}(\Omega)}^{2}\right)
\end{aligned}
$$

holds with the constant $C$ independent of $u, f, g_{j}$ and $t_{0}$.

Proof. Without generality we assume that the domain $\Omega$ coincides with the cone $K$ in the unit ball. Set $\Omega^{0}=\left\{x \in \Omega:|x| \geq 2^{-1}\right\}$,

$$
\Omega^{k}=\left\{x\left|x \in \Omega, 2^{-k} \leq\right| x \mid \leq 2^{-k+1}\right\}, \quad k=1,2, \ldots,
$$

and $\Gamma^{k}=\partial \Omega \cap \partial \Omega^{k}, k=0,1 \ldots$ According to well known results on the regularity of solutions of elliptic boundary problems in smooth domains (see, e.g., [12]), we have 


$$
\begin{aligned}
\|u\|_{H^{l+2 m}\left(\Omega^{2}\right)}^{2} \leq & C\left(\|f\|_{H^{l}\left(\Omega^{1} \cup \Omega^{2} \cup \Omega^{3}\right)}^{2}+\|u\|_{L_{2}\left(\Omega^{1} \cup \Omega^{2} \cup \Omega^{3}\right)}^{2}\right) \\
& +\left\|g_{j}\right\|_{W^{l+j-}}^{2} \frac{1}{2}{ }_{\left(\Gamma^{1} \cup \Gamma^{2} \cup \Gamma^{3}\right)}
\end{aligned}
$$

with the constant $C$ independent of $u, f, g_{j}$ and $t_{0}$. By making change of variable $x=$ $2^{-k} x^{\prime}$ for a positive integer $k$, we get from (4.3), (4.4) that

$$
\begin{aligned}
& \sum_{|p|=2 m} a_{p} D_{x^{\prime}}^{p} u+\sum_{|p| \leq 2 m-1} 2^{(|p|-2 m) k} a_{p} D_{x^{\prime}}^{p} u=2^{-2 m k} f \text { in } \Omega, \\
& \sum_{|p|=2 m-j} b_{j p} D_{x^{\prime}}^{p} u+\sum_{|p| \leq 2 m-j-1} 2^{(|p|-2 m+j) k} b_{j p} D_{x^{\prime}}^{p} u=2^{-(2 m-j) k} g_{j} \quad \text { on } \Gamma, \quad j=1, \ldots, m .
\end{aligned}
$$

Similarly as above, from (4.6), (4.7), we have

$$
\begin{aligned}
\|u\|_{W^{l+2 m}\left(\Omega^{2}\right)}^{2} \leq C & \left(\left\|2^{-2 m k} f\right\|_{W^{l}\left(\Omega^{1} \cup \Omega^{2} \cup \Omega^{3}\right)}^{2}\right. \\
& \left.+\left\|2^{-(2 m-j) k} g_{j}\right\|_{W}^{2}{ }_{W}^{l+j-} \frac{1}{2}{ }_{\left(\Gamma^{1} \cup \Gamma^{2} \cup \Gamma^{3}\right)}+\|u\|_{L_{2}\left(\Omega^{1} \cup \Omega^{2} \cup \Omega^{3}\right)}^{2}\right)
\end{aligned}
$$

with the constant $C$ independent of $u, f, g_{j}, t_{0}$ and $k$. Let $\tilde{g}_{j} \in V_{2, \alpha}^{l+j}(\Gamma)$ be arbitrary extensions of $g_{j}$ to $\Omega, j=1, \ldots, m$. Then we have from (4.8) that

$$
\begin{aligned}
\|u\|_{W^{l+2 m}\left(\Omega^{2}\right)}^{2} \leq C & \left\|2^{-2 m k} f\right\|_{W^{l}\left(\Omega^{1} \cup \Omega^{2} \cup \Omega^{3}\right)}^{2} \\
& \left.+\left\|2^{-(2 m-j) k} \tilde{g}_{j}\right\|_{W^{l+j}\left(\Omega^{1} \cup \Omega^{2} \cup \Omega^{3}\right)}^{2}+\|u\|_{L_{2}\left(\Omega^{1} \cup \Omega^{2} \cup \Omega^{3}\right)}^{2}\right) .
\end{aligned}
$$

Returning to variable $x$ with noting that, in $\Omega^{k+2}, 2^{-k-2} \leq r \leq 2^{-k-1}$, from (4.9) we have

$$
\begin{aligned}
& \sum_{|p| \leq l+2 m}\left\|r^{2(p+|p|-l-2 m)} u\right\|_{L\left(\Omega^{k+2}\right)}^{2} \leq C\left(\sum_{|p| \leq l}\left\|r^{2(p+|p|-l)} f\right\|_{L\left(\Omega^{k+1} \cup \Omega^{k+2} \cup \Omega^{k+3}\right)}^{2}\right. \\
& \left.+\sum_{|p| \leq l+j}\left\|r^{2(p+|p|-l-j)} g_{j}\right\|_{W^{l+j}\left(\Omega^{k+1} \cup \Omega^{k+2} \cup \Omega^{k+3}\right)}^{2}+\left\|r^{2(p-l-2 m)} u\right\|_{L_{2}\left(\Omega^{k+1} \cup \Omega^{k+2} \cup \Omega^{k+3}\right)}^{2}\right) .
\end{aligned}
$$

Taking sum both sides of these inequalities with respect to $k$ from 1 to $\infty$, we have

$$
\|u\|_{V_{2, \alpha}^{l+2 m}(\Omega)}^{2} \leq C\left(\|f\|_{V_{2, \alpha}^{l}(\Omega)}^{2}+\sum_{j=1}^{m}\left\|\tilde{g}_{j}\right\|_{V_{2, \alpha}^{l+j+1}(\Omega)}^{2}+\|u\|_{V_{2, \alpha-l-2 m}^{0}(\Omega)}^{2}\right) .
$$

Here it is noted that $\sum_{|p| \leq l+2 m}\left\|r^{2(\alpha+|p|-l-2 m)} u\right\|_{L\left(\Omega^{0}\right)}^{2}$ can be estimated by the right-hand side of (4.11). It follows from (4.11) that

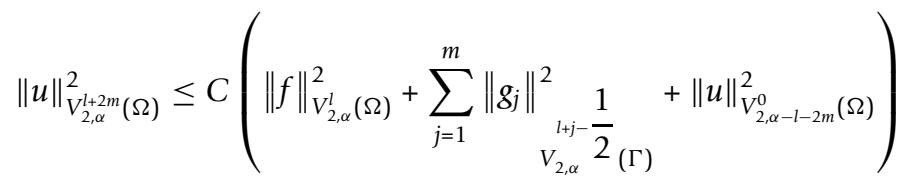

with the constant $C$ independent of $u, f, g_{j}$ and $t_{0}$. 
Lemma 4.3. Let $t_{0}$ be a fixed number in $[0, \infty), u \in H_{l o c}^{2 m}(\bar{\Omega} \backslash\{0\}) \cap H^{m}(\Omega)$ be a generalized solution of the following elliptic boundary value problem

$$
\begin{aligned}
& L\left(x, t_{0}, D\right) u=f \quad \text { in } \Omega, \\
& N_{j}\left(x, t_{0}, D\right) u=g_{j} \quad \text { on } \Gamma, \quad j=1, \ldots, m,
\end{aligned}
$$

where $f \in H_{m+\varepsilon}^{0}(\Omega), \quad \underset{g_{j} \in H_{m+\varepsilon}-\frac{1}{2}}{(\Gamma)^{\prime}}, j=1, \ldots, m, \varepsilon$ is defined as in Theorem 4.1. Then $u \in H_{m+\varepsilon}^{2 m}(\Omega)$. Moreover, the following inequalities

$$
\|u\|_{H_{m+\varepsilon}^{2 m}(\Omega)}^{2} \leq C\left(\|f\|_{H_{m+\varepsilon}^{0}(\Omega)}^{2}+\sum_{j=1}^{m}\left\|g_{j}\right\|_{\substack{j-\frac{1}{H_{m+\varepsilon}}(\Gamma) \\ 2}}^{1}+\|u\|_{H^{m}(\Omega)}^{2}\right),
$$

holds with the constant $C$ independent of $u$, and $f, g_{j}$, and $t_{0}$.

Proof. Firstly, since $m+\varepsilon>j+1-\frac{n}{2}$ for $j=1, \ldots, m$, by (2.3), it holds that

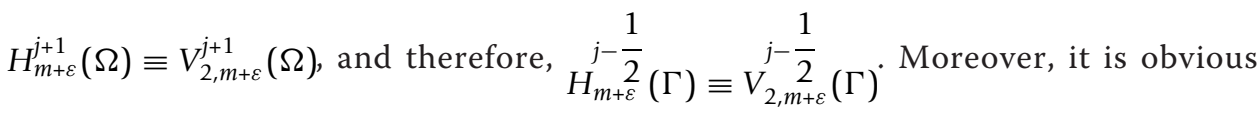
that $H_{m+\varepsilon}^{0}(\Omega) \equiv V_{2, m+\varepsilon}^{0}(\Omega)$. Hence, $f \in V_{2, m+\varepsilon}^{0}(\Omega)$ and $g_{j} \in V_{2, m+\varepsilon}^{j-\frac{1}{2}}(\Gamma)$ for $j=1, \ldots, m$.

If $m<\frac{n}{2}$, then $H^{m}(\Omega) \equiv H_{0}^{m}(\Omega) \equiv V_{2,0}^{m}(\Omega) \subset V_{2,-m}^{0}(\Omega)$ by (2.3) and (2.2). Thus the assertion of the lemma follows from Lemma 4.2 with noting that the space $V_{2, m+\varepsilon}^{2 m}(\Omega)$ is continuously imbedded in $H_{m+\varepsilon}^{2 m}(\Omega)$ according to (2.1).

Now consider the case $m \geq \frac{n}{2}$. Let $\ell=\left[\frac{n}{2}\right]$ be the greatest integer not exceeding $\frac{n}{2}$. Then we have $\ell-\frac{n}{2}<\varepsilon<\ell+1-\frac{n}{2}$. According to [[2], Th. 7.1.1], the function $u$ which belongs to $H^{m}(\Omega) \subset H_{\varepsilon}^{m}(\Omega)$ has the representation

$$
u(x)=\sum_{|\alpha| \leq m-\ell-1} c_{\alpha} x^{\alpha}+v(x)
$$

where $v \in V_{2, \varepsilon}^{m}(\Omega)$, and

$$
c_{\alpha}=\frac{1}{\alpha !} \lim _{r \rightarrow 0} \frac{1}{|\Omega|} \int_{\Omega} D_{x}^{\alpha} u(\omega, r) d \omega
$$

with the following estimates

$$
\begin{aligned}
& \|v\|_{V_{2, \varepsilon}^{m}(\Omega)} \leq C\|u\|_{H^{m}(\Omega)} \\
& \left|c_{\alpha}\right| \leq C\|u\|_{H^{m}(\Omega),}|\alpha| \leq m-\ell-1 .
\end{aligned}
$$

Here $|\Omega|=\int_{\Omega} d \omega$, and $C$ is a constant independent of $u$ and $t_{0}$. Put $w=\sum_{|\alpha| \leq m-\ell-1} c_{\alpha} x^{\alpha}$. From (4.16) we see easily that $w \in H_{m+\varepsilon}^{2 m}(\Omega)$ and

$$
\|w\|_{H_{m+\varepsilon}^{2 m}(\Omega)} \leq C\|u\|_{H^{m}(\Omega)}
$$


where $C$ is a constant independent of $u$ and $t_{0}$. From this we have

$$
\begin{aligned}
& L\left(x, t_{0}, D\right) w \in H_{m+\varepsilon}^{0}(\Omega)=V_{2, m+\varepsilon}^{0}(\Omega), \\
& N_{j}\left(x, t_{0}, D\right) w \in H_{m+\varepsilon}^{j-\frac{1}{2}}(\Gamma)=V_{2, m+\varepsilon}^{j-\frac{1}{2}}(\Gamma) .
\end{aligned}
$$

Hence, it follows from (4.12) and (4.13) that

$$
\begin{aligned}
& L\left(x, t_{0}, D\right) v=\tilde{f}:=f-L\left(x, t_{0}, D\right) w \in V_{2, m+\varepsilon}^{0}(\Omega), \\
& N_{j}\left(x, t_{0}, D\right) v=\tilde{g}_{j}:=g_{j}-N_{j}\left(x, t_{0}, D\right) w \in V_{2, m+\varepsilon}^{j-\frac{1}{2}}(\Gamma), \quad j=1, \ldots, m .
\end{aligned}
$$

Now we can apply Lemma 4.2 to conclude from (4.17) and (4.18) that $v \in V_{2, m+\varepsilon}^{2 m}(\Omega)$. Therefore, $u=v+w \in H_{m+\varepsilon}^{2 m}(\Omega)$ with the estimate (4.14). The lemma is completely proved.

Proof of Theorem 4.1: First, we show by induction on $h$ that

$$
u_{t^{k}}(\cdot, t) \in H_{l o c}^{2 m}(\bar{\Omega} \backslash\{0\}) \quad \text { for a.e. } t \in(0, \infty) \quad \text { and all } k \leq h-1 .
$$

According to Theorem 3.4 it holds that $u_{t^{k}} \in H^{m, 1}\left(Q_{1}(k+1) \gamma+\sigma\right), k \leq h$. In particular, $u_{t t} \in L_{2}(Q, 2 \gamma+\sigma)$. Thus, from the equality (2.9) it follows that

$$
B(t, u, \eta)=\left(f(\cdot, t)-u_{t t}(\cdot, t), \eta\right)
$$

for all $\eta \in H^{m}(\Omega)$ and a.e. $t \in(0, \infty)$. Since $f(\cdot, t)-u_{t t}(\cdot, t) \in L_{2}(\Omega)$ for a.e. $t \in(0$, $\infty)$, according to results for elliptic boundary value problem in domains with smooth boundaries, it follows from (4.20) that $u(\cdot, t) \in H_{l o c}^{2 m}(\bar{\Omega} \backslash\{0\})$ for a.e. $t \in(0, \infty)$, moreover, the function $u$ satisfies the following equalities:

$$
L(x, t, D) u=f-u_{t t}
$$

for a.e. $(x, t) \in Q$ and

$$
N_{j}(x, t, D) u=0 \quad \text { on } S
$$

in the trace sense. Thus, the assertion (4.19) holds for $h=1$, and by (2.5) we also have

$$
(L(x, t, D) u, \eta)=B(t, u, \eta)
$$

for all $\eta \in C_{0}^{\infty}(\bar{\Omega} \backslash\{0\})$ and a.e. $t \in(0, \infty)$. Assume now (4.19) holds for $h$ - 2. It follows from (4.20) that

$$
B\left(t, u_{t^{h-1}}, \eta\right)=\left(f_{t^{h-1}}(\cdot, t), \eta\right)-\left(u_{t^{h+1}}(\cdot, t), \eta\right)-\sum_{k=0}^{h-2}\left(\begin{array}{c}
h-1 \\
k
\end{array}\right) B_{t^{h-1-k}}\left(t, u_{t^{k}}, \eta\right)
$$

for all $\eta \in H^{m}(\Omega)$, a.e. $t \in(0, \infty)$. Since $u_{t^{k}} \in H_{l o c}^{2 m}(\bar{\Omega} \backslash\{0\})$, by the induction assumption, we have from (4.21) that

$$
\left(L_{t^{h-1-k}}(x, t, D) u, \eta\right)=B_{t^{h-1-k}}(t, u, \eta) \quad(k \leq h-2)
$$


for all $\eta \in C_{0}^{\infty}(\bar{\Omega} \backslash\{0\})$ and a.e. $t \in(0, \infty)$. Combining (4.22) and (4.23) we obtain

$$
B\left(t, u_{t^{h-1}}, \eta\right)=\left(F_{h-1}(\cdot, t), \eta\right)
$$

for all $C_{0}^{\infty}(\bar{\Omega} \backslash\{0\})$ and a.e. $t \in(0, \infty)$, where

$$
F_{h-1}=f_{t^{h-1}}(\cdot, t)-u_{t^{h+1}}(\cdot, t)-\sum_{k=0}^{h-2} h-1 k L_{t^{h-1-k}}(\cdot, t) u_{t^{k}} \in L_{2, l o c}(\bar{\Omega} \backslash\{0\}) .
$$

Similarly as above, it follows from (4.24) that $u_{t^{h}}(\cdot, t) \in H_{l o c}^{2 m}(\bar{\Omega} \backslash\{0\})$ for a.e. $t \in(0$, $\infty)$, and therefore, (4.19) holds for $h-1$.

Now we prove the assertion of the theorem by induction on $h$. Let us consider first the case $h=1$. We rewrite (2.6), (2.7) in the form

$$
\begin{aligned}
& L(x, t, D) u=f_{1}:=f-u_{t t} \quad \text { in } Q, \\
& N_{j}(x, t, D) u=0 \quad \text { on } S, \quad j=1, \ldots, m .
\end{aligned}
$$

Since $f_{1}(\cdot, t) \in L_{2}(\Omega) \subset H_{\alpha}^{0}(\Omega)$ for a.e. $t \in[0, \infty)$, by Lemma 4.3, it follows from (4.25) and (4.26) that $u(\cdot, t) \in H_{m+\varepsilon}^{2 m}(\Omega)$ for a.e. $t \in(0, \infty)$ and

$$
\|u(\cdot, t)\|_{H_{m+\varepsilon}^{2 m}(\Omega)}^{2} \leq C\left(\left\|f_{1}(\cdot, t)\right\|_{L_{2}(\Omega)}^{2}+\|u(\cdot, t)\|_{H^{m}(\Omega)}^{2}\right),
$$

where $C$ is a constant independent of $u, f_{1}$ and $t$. Since the trip

$$
m-\varepsilon-\frac{n}{2} \leq \operatorname{Re} \lambda \leq 2 m-\alpha-\frac{n}{2}
$$

does not contain any eigenvalue of $\mathcal{U}(\lambda, t)$ for all $t \in[0,+\infty)$, and $\varepsilon+\frac{n}{2} \notin\{1, \ldots, m\}$ by the definition of the number $\varepsilon$, we can apply Theorem 7.2.4 and the note below Theorem 7.3.5 of [2] to conclude from (4.25), (4.26) that $u(\cdot, t) \in H_{\alpha}^{2 m}(\Omega)$ and

$$
\|u(\cdot, t)\|_{H_{\alpha}^{2 m}(\Omega)}^{2} \leq C\left(\left\|f_{1}(\cdot, t)\right\|_{L_{2}(\Omega)}^{2}+\|u(\cdot, t)\|_{H^{m}(\Omega)}^{2}\right),
$$

where $C$ is a constant independent of $u, f_{1}$ and $t$. Now multiplying both sides of (4.27) with $e^{-2(2 \gamma+\sigma) t}$, then integrating with respect to $t$ from 0 to $\infty$ and using estimates from Theorem 3.4, we obtain

$$
\|u\|_{H_{\alpha}^{2 m, 1}(Q, 2 \gamma+\sigma)}^{2} \leq C\|f\|_{L_{2}(Q, \sigma)^{\prime}}^{2}
$$

where $C$ is a constant independent of $u$ and $f$. Hence, the theorem is valid for $h=1$. Assume that the theorem is true for some nonnegative $h-2$. We will prove it for $h$ 1. Differentiating $(h-1)$ times both sides of (4.25), (4.26) with respect to $t$, we have

$$
\begin{aligned}
& L u_{t^{h-1}}=\tilde{f}:=f_{t^{h-1}}-u_{t^{h+1}}-\sum_{k=0}^{h-2}\left(\begin{array}{c}
h-1 \\
k
\end{array}\right) L_{t^{h-1-k}} u_{t^{k}} \quad \text { in } Q, \\
& N_{j} u_{t^{h-1}}=\tilde{g}_{j}:=-\sum_{k=0}^{h-2}\left(\begin{array}{c}
h-1 \\
k
\end{array}\right)\left(N_{j}\right)_{t^{h-1-k}} u_{t^{k}} \quad \text { on } S, \quad j=1, \ldots, m .
\end{aligned}
$$


By the induction assumption, it holds that

$$
u_{t^{k}} \in H_{\alpha}^{2 m, 1}(Q,(k+2) \gamma+\sigma) \subset H_{\alpha}^{2 m, 1}(Q,(h+1) \gamma+\sigma), k=0, \ldots, h-2 .
$$

Moreover,

$$
f_{t^{h-1}} \in L_{2}(Q,(h-1) \gamma+\sigma) \subset L_{2}(Q,(h+1) \gamma+\sigma)
$$

by the assumption of the theorem and

$$
u_{t^{h+1}} \in L_{2}\left(Q_{,}(h+1) \gamma+\sigma\right)
$$

by Theorem 3.4. Thus, for a.e. $t \in(0, \infty)$, we have $\tilde{f}(\cdot, t) \in H_{\alpha}^{0}(\Omega), \quad \tilde{g}(\cdot, t) \in H_{\alpha}^{j-} \frac{1}{2}(\Gamma)$ and

$$
\begin{aligned}
&\|\tilde{f}(\cdot, t)\|_{H_{\alpha}^{0}(\Omega)}^{2} \leq C\left(\left\|f_{t^{h-1}}(\cdot, t)\right\|^{2}+\left\|u_{t^{h+1}}(\cdot, t)\right\|_{L_{2}(\Omega)}^{2}+\sum_{k=0}^{h-2}\left\|u_{t^{k}}(\cdot, t)\right\|_{H_{\alpha}^{2 m}(\Omega)}^{2}\right) . \\
&\left\|\tilde{g}_{j}(\cdot, t)\right\|_{j-\frac{1}{2}}^{2} \leq C \sum_{k=0}^{h-2}\left\|u_{t^{k}}(\cdot, t)\right\|_{H_{\alpha}^{2 m}(\Omega)}^{2}, j=1, \ldots, m,
\end{aligned}
$$

where $C$ is the constant independent of $u, f$ and $t$. Now we can repeat the arguments above to conclude that $u_{t^{h-1}} \in H_{\alpha}^{2 m, 1}(Q,(h+1) \gamma+\sigma)$ with the estimate (4.2) for $k=h$ -1 . The proof is completed.

\section{An example}

In this section we apply the previous results to the Cauchy-Neumann problem for the classical wave equation. We consider the following problem:

$$
\begin{aligned}
& u_{t t}-\Delta u=f \text { in } Q, \\
& \frac{\partial u}{\partial v}=0 \text { on } S, \\
& \left.u\right|_{t=0}=\left.u_{t}\right|_{t=0}=0 \text { on } \Omega,
\end{aligned}
$$

where $\Delta$ is the Laplace operator.

For problem (5.1)-(5.3) it can be directly verified that the constants $\mu, \mu_{1}$ and $\gamma_{0}$ are now defined by

$$
\mu=1, \mu_{1}=0 \text { and } \gamma_{0}=0 .
$$

The operator pencil associated with the problem (5.1)-(5.3) is now defined by (see [[13], Sec. 2.3])

$$
\mathcal{U}(\lambda, t) u=\mathcal{U}(\lambda) u \mid=\left(\delta u+\lambda(\lambda+n-2) u,\left.\partial_{\nu} u\right|_{\partial G}\right),
$$

where $\delta$ is the Laplace-Beltrami operator on the unit sphere $S^{n-1}$. It is well known that (see also [[13], Sec. 2.3]) the trip

$$
2-n<\operatorname{Re} \lambda<0
$$


does not contains any eigenvalue of the operator pencil $\mathcal{U}(\lambda, t)$. We see that if $0 \leq \alpha$ $\leq 1$ and $n>4-2 \alpha$, then the trip (4.1) with $m=1$ is contained in the trip (5.5), since $\varepsilon$ can be chosen as zero or an arbitrary small positive real number. Thus, we can apply Theorem 4.1 to receive the following result.

Theorem 5.1. Let $h$ be a nonnegative integer and $\alpha$ be a real number, $0 \leq \alpha \leq 1$. Assume that the vector function $f$ satisfy the following conditions for some nonnegative real number $\sigma$

$$
\begin{aligned}
& \text { (i) } f_{t^{k}} \in L_{2}(Q, \sigma), k \leq h, \\
& \text { (ii) } f_{t^{k}}(x, 0)=0,0 \leq k \leq h-1 .
\end{aligned}
$$

Assume further that $n>4-2 \alpha$. Then for an arbitrary positive real number $\gamma$ the problem (5.1)-(5.3) has a unique generalized solution $u$ in the space $H^{1,1}(Q, \gamma+\sigma)$ which has derivatives with respect to $t$ up to the order $h$ with $u_{t^{k}} \in H_{\alpha}^{2,1}(Q,(k+2) \gamma+\sigma)$ for $k$ $=0,1, \ldots, h-1$, and

$$
\sum_{k=0}^{h-1}\left\|u_{t^{k}}\right\|_{H_{\alpha}^{2,1}(Q,(k+2) \gamma+\sigma)}^{2} \leq C \sum_{k=0}^{h}\left\|f_{t^{k}}\right\|_{L_{2}(Q, \sigma)}^{2},
$$

where $C$ is a constant independent of $u$ and $f$.

\section{Acknowledgements}

This work was supported by National Foundation for Science and Technology Development (NAFOSTED), Vietnam, under project no. 101.01.58.09.

\section{Author details}

${ }^{1}$ Department of Mathematics, Hanoi National University of Education, Hanoi, Vietnam ${ }^{2}$ Department of Mathematics, Can Tho University, Can Tho City, Vietnam

\section{Authors' contributions}

All authors typed, read and approved the final manuscript.

\section{Competing interests}

The authors declare that they have no competing interests.

Received: 22 January 2011 Accepted: 25 August 2011 Published: 25 August 2011

\section{References}

1. Kondrat'ev, VA, Oleinik, OA: Boundary value problems for partial differential equations in nonsmooth domains. Usp Math Nauka. 38(2), 1-86 (1983)

2. Kozlov, VA, Maz'ya, VG, Rossmann, J: Elliptic Boundary Problems in Domains With Point Singularities, Mathematical Surveys and Monographs vol. 85. American Mathematical Society, Providence, Rhode Island. (1997)

3. Hung, NM: Asymptotic behaviour of solutions of the first boundary-value problem for strongly hyperbolic systems near a conical point at the boundary of the domain. Math Sb 190(7), 103-126 (1999). (in Russian). Trans. in Sb. Math. 190 (78), 1035-1058 (1999)

4. Hung, NM, Kim, BT: On the solvability of the first mixed problem for strongly hyperbolic system in infinite nonsmooth cylinders. Taiwanese J Math. 12(9), 2601-2618 (2008)

5. Hung, NM, Yao, JC: Cauchy-Dirichlet problem for second-order hyperbolic equations in cylinder with non-smooth base. Nonlinear Anal TMA. 70, 741-756 (2009). doi:10.1016/j.na.2008.01.007

6. Kokotov, A, Plamenevskii, BA: On the asymptotic on solutions to the Neumann problem for hyperbolic systems in domain with conical point. St. Petersburg Math J. 16(3), 477-506 (2005)

7. Renardy, M, Rogers, RC: An Introduction to Partial Differential Equations. Springer, New York, 2 (2004)

8. Hale, J: Theory of Functional Differential Equations. Springer, New York (1977)

9. Adams, RA: Sobolev Spaces. Academic Press, London (1975)

10. Evans, LC: Partial Differential Equations, Graduate Studies in Mathematics vol. 19. American Mathematical Society, Providence, Rhode Island. (1998)

11. Hung, NM, Anh, NT: The initial-boundary value problems for parabolic equations in domains with conical points. In: Albert, R, Baswell (eds.) Advances in Mathematics Research, vol. 10, Nova Science Publishers, Inc, New York, USA (2009)

12. Agmon, S, Douglis, A, Nirenberg, L: Estimates near the boundary for solutions of elliptic partial differential equations satisfying general boundary conditions I. Commun Pure Appl Math. 12, 623-727 (1959). doi:10.1002/cpa.3160120405 
13. Kozlov, VA, Maz'ya, VG, Rossmann, J: Spectral Problems Associated With Corner Singularities Of Solutions to Elliptic Equations. In Mathematical Surveys and Monographs, vol. 85,American Mathematical Society, Providence, Rhode Island (2001)

doi:10.1186/1687-2770-2011-17

Cite this article as: Hung et al: On the regularity of the solution for the second initial boundary value problem for hyperbolic systems in domains with conical points. Boundary Value Problems 2011 2011:17.

Submit your manuscript to a SpringerOpen ${ }^{\odot}$ journal and benefit from:

- Convenient online submission

- Rigorous peer review

- Immediate publication on acceptance

- Open access: articles freely available online

- High visibility within the field

- Retaining the copyright to your article

Submit your next manuscript at $\gg$ springeropen.com 